\title{
The surface catalytic mechanism: a comparative study with square-wave and staircase cyclic voltammetry
}

Received: 23 November 2001 / Accepted: 23 April 2002 / Published online: 4 July 2002

(C) Springer-Verlag 2002

\begin{abstract}
A pseudo-first-order catalytic mechanism in which both reactant and product of a redox reaction are strongly immobilized on an electrode surface is theoretically analysed under conditions of square-wave (SWV) and staircase cyclic voltammetry (SCV). A mathematical procedure is developed under diffusionless conditions. The relationships between the properties of the voltammetric response and both the kinetic parameters of the redox reaction and the parameters of the excitation signal are studied. The phenomenon of the quasi-reversible maximum is discussed. A comparative study between SWV and SCV is presented and the limitations and advantages of both techniques, from analytical and kinetic points of view, are discussed. The theoretical predictions are experimentally confirmed by the redox reaction of azobenzene in the presence of hydrogen peroxide as an oxidizing agent.
\end{abstract}

Keywords Square-wave voltammetry Staircase cyclic voltammetry $\cdot$ Surface catalytic mechanism $\cdot$ Azobenzene

\section{Introduction}

If both components of a redox couple are firmly immobilized on a working electrode surface, and if

V. Mirčeski $(\bowtie) \cdot$ R. Gulaboski

Ernst-Moritz-Arndt-Universität Greifswald,

Institut für Chemie und Biochemie,

Soldtmannstrasse 23,

17489 Greifswald, Germany

E-mail: valentinmirceski@netscape.net

Tel.: + 49-3834-864439

Fax: + 49-3834-864451

V. Mirčeski

Institute of Chemistry,

Faculty of Natural Sciences and Mathematics,

"Sv. Kiril i Metodij" University,

P.O. Box 162, 1000 Skopje, Republic of Macedonia diffusional mass transport is negligible in the course of the electrode reaction, then the redox reaction occurs predominantly at the electrode surface, and the entire electrode mechanism is designated as a surface redox reaction $[1,2,3,4,5,6,7]$. Such processes form an important class of redox reactions observed frequently with conducting and redox polymer films, self-assembled structures populated with redox sites, and simple adsorbates. The knowledge of their thermodynamic and kinetic properties, collected by exploiting various voltammetric methods, is of considerable importance for surface science.

The theoretical study of surface redox reactions was initiated by Laviron [8,9] by applying cyclic linear scan voltammetry (CV). Nowadays the applicability of Laviron's theory is restricted appreciably, since in many modern electrochemical systems the linear potential scan is replaced by staircase potential ramps during the cyclic voltammetric experiment. It should be pointed out that staircase cyclic voltammetry (SCV) $[10,11,12,13,14,15,16]$ is equivalent to classical $\mathrm{CV}$ in a limiting case only, e.g. a short duration of potential treads and small scan increments $[11,13,14$, 15]. For these reasons, the response of many redox systems differs under conditions of $\mathrm{CV}$ and SCV. In particular, the discrepancies are considerable when adsorption is involved in the electrode reaction, and especially for surface redox reactions [17]. For all these reasons, various surface electrode mechanisms deserve further theoretical consideration under conditions of SCV.

Although surface redox processes have been known for a long time, interest in their study by square-wave voltammetry (SWV) has recently been renewed $[1,2,3$, $4,5,6,7]$. This technique, which comprises the advantages of pulse voltammetric techniques and cyclic voltammetry, appears to be a suitable method for redox kinetic measurements of adsorption coupled redox reactions $[18,19,20,21,22,23]$.

We have recently shown that the SW response of a simple surface redox reaction may be significantly en- 
hanced in the presence of an oxidizing agent (OA) [24]. Such an electrode mechanism, in which the oxidized form of the redox couple is regenerated by means of a chemical reaction, is designated as a catalytic surface electrode reaction (Eqs. 1 and 2):

$$
\begin{aligned}
& \mathrm{Ox}_{(\mathrm{ads})}+n \mathrm{e}^{-} \leftrightarrows \operatorname{Red}_{(\mathrm{ads})} \\
& \operatorname{Red}_{(\mathrm{ads})}+\mathrm{OA} \rightarrow \mathrm{Ox}_{(\mathrm{ads})}+\mathrm{P}
\end{aligned}
$$

Here, Ox and Red are the oxidized and reduced forms of the redox couple, respectively, and $\mathrm{P}$ is a certain electroinactive product. Surface catalytic redox reactions are important because they provide the possibility to improve the sensitivity of electroanalytical methods $[25,26$, 27, 28, 29, 30]. Moreover, these electrode reactions are widely exploited for the development of amperometric sensors [31, 32, 33].

In this paper, we apply the previous theoretical treatment [24] to the study of the surface catalytic mechanism, applying both staircase cyclic and square-wave voltammetry. The combination of these two techniques is a powerful tool for mechanistic, thermodynamic, and redox kinetic characterization of the mechanism under study. Besides, the aim of the work is to elucidate the advantages and limitations of both techniques, as well as to clarify the range of applicability of each technique for an estimation of the kinetic parameters of the investigated redox system.

\section{Theoretical model}

A pseudo-first-order surface catalytic mechanism, in which both species of the redox couple are strongly immobilized on the electrode surface, is considered (Eqs. 1 and 2). It is assumed that the reaction is controlled by charge transfer kinetics (Eq. 1) as well as by the kinetics of the pseudo-first-order "catalytic" reaction (Eq. 2). Furthermore, it is assumed that adsorption of both the reactant and product of the redox reaction is totally irreversible and there are no significant interactions between the adsorbed species in the case of a submonolayer surface coverage. An additional condition is that in the course of the voltammetric measurements the adsorption and redox reactions of the dissolved molecules can be neglected. Thus, the above electrode mechanism is represented by the following mathematical model:

$$
\begin{aligned}
& \mathrm{d} \Gamma_{\text {Ox }} / \mathrm{d} t=-\frac{I}{n F S}+k_{\mathrm{c}} \Gamma_{\text {Red }} \\
& \mathrm{d} \Gamma_{\text {Red }} / \mathrm{d} t=\frac{I}{n F S}-k_{\mathrm{c}} \Gamma_{\text {Red }} \\
& t=0: \Gamma_{\text {Ox }}=\Gamma_{0}, \quad \Gamma_{\text {Red }}=0 \\
& t>0: \Gamma_{\text {Ox }}+\Gamma_{\text {Red }}=\Gamma_{0}
\end{aligned}
$$

where $\Gamma_{\mathrm{Ox}}$ and $\Gamma_{\mathrm{Red}}$ are surface concentrations of $\mathrm{Ox}$ and Red species, respectively, while $\Gamma_{0}$ is the initial concentration of Ox species. $k_{\mathrm{c}}$ is the pseudo-first-order "catalytic" rate constant of the reaction (2) expressed in unit of s ${ }^{-1}$. It is defined as $k_{\mathrm{c}}=k_{\mathrm{c}}{ }^{\prime} c(\mathrm{OA})$ where $k_{\mathrm{c}}{ }^{\prime}$ is the real rate constant of the "catalytic" reaction in units of $\mathrm{s}^{-1} \mathrm{~mol}^{-1} \mathrm{~L}$, while $c(\mathrm{OA})$ is the concentration of the oxidising agent $\mathrm{OA}$, present in a large excess.

The solutions of equations (3) and (4), representing the variation of the surface concentrations of Ox and Red with time during the single potential pulse, are:

$\Gamma_{\text {Ox }}=\Gamma_{0}-\int_{0}^{t} \frac{I(\tau)}{n F S} \exp \left(-k_{\mathrm{c}}(t-\tau)\right) \mathrm{d} \tau$

and

$\Gamma_{\text {Red }}=\int_{0}^{t} \frac{I(\tau)}{n F S} \exp \left(-k_{\mathrm{c}}(t-\tau)\right) \mathrm{d} \tau$

In addition, at the electrode surface, the following condition holds:

$\frac{I}{n F S}=k_{\mathrm{s}} \exp (-\alpha \phi)\left(\Gamma_{\text {Ox }}-\exp (\phi) \Gamma_{\text {Red }}\right)$

Here, $k_{\mathrm{s}}$ is the standard rate constant of the redox reaction expressed in unit of $\mathrm{s}^{-1}, \alpha$ is the cathodic transfer coefficient, and $\phi$ is the relative dimensionless electrode potential defined as $\phi=n F\left(E-E^{\circ}\right) / R T$. Substituting Eqs. 5 and 6 into Eq. 7, Eq. 8 is obtained:

$$
\begin{aligned}
\frac{I}{n F S}= & k_{\mathrm{s}} \exp (-\alpha \phi) \Gamma_{0}-k_{\mathrm{s}} \exp (-\alpha \phi)(1+\exp (\phi)) \\
& \times \int_{0}^{t} \frac{I(\tau)}{n F S} \exp \left(-k_{\mathrm{c}}(t-\tau)\right) \mathrm{d} \tau
\end{aligned}
$$

This last equation is the mathematical representation of the surface catalytic mechanism under voltammetric conditions.

The numerical solution of Eq. 8 was obtained by the method of Nicholson and Olmstead [34]. Both variables, the dimensionless current $\Psi$ and time $t$, were incremented. To each time $t=m d$, where $d$ is the time increment, a certain current $\Psi_{m}$ was ascribed. The numerical solution is represented by the following set of recursive formulae:

$$
\begin{aligned}
& \Psi_{1}=\frac{\lambda \exp \left(-\alpha \phi_{1}\right)}{1+\lambda \exp \left(-\alpha \phi_{1}\right)\left(1+\exp \left(\phi_{1}\right)\right) \frac{M_{1}}{\gamma}} \\
& \Psi_{m}=\frac{\lambda \exp \left(-\alpha \phi_{m}\right)\left(1-\frac{1+\exp \left(\phi_{m}\right)}{\gamma} \sum_{j=1}^{m-1} \Psi_{j} M_{m-j+1}\right)}{1+\lambda \exp \left(-\alpha \phi_{m}\right)\left(1+\exp \left(\phi_{m}\right)\right) \frac{M_{1}}{\gamma}}
\end{aligned}
$$

The numerical solution formally has an equivalent form for both techniques. Nevertheless, the dimension- 
less parameters are defined differently for each technique. For SWV the following definitions hold: $\Psi_{m}=$ $I_{m} / n F S f \Gamma_{0}$ is the dimensionless current, $\lambda=k_{\mathrm{s}} / f$ is the redox kinetic parameter, $\gamma=k_{\mathrm{c}} / f$ is the catalytic parameter, $M_{m}=\exp \left(-\frac{\gamma}{50}(m-1)\right)-\exp \left(-\frac{\gamma}{50}(m)\right)$ is the numerical integration factor, and $f$ is the SW frequency. During the SWV experiment, a square-wave excitation signal is applied to the working electrode. This excitation signal is a train of cathodic and anodic pulses superimposed on a staircase potential ramp and characterized by amplitude $E_{\mathrm{sw}}$, which is half of the peak-to-peak height, the frequency of pulses $f$, and the scan increment $\mathrm{d} E$ of the staircase potential ramp.

For staircase $\mathrm{CV}$ the following definitions are valid: $\Psi_{m}=\frac{I_{m} \tau}{n F S \Gamma_{0}}$ is the dimensionless current, $\gamma=k_{\mathrm{c}} \tau$ is the dimensionless catalytic parameter, $\lambda=k_{\mathrm{s}} \tau$ is the dimensionless redox kinetic parameter, and $\tau$ is the duration of the single potential tread. The numerical integration factor $M$ is defined as $M_{m}=\exp \left(-\frac{\gamma}{25}(m-1)\right)-\exp \left(-\frac{\gamma}{25}(m)\right)$. The excitation signal used in SCV consists only of a staircase potential ramp, which is characterized by the duration of the potential tread $\tau$ and the step of the staircase ramp $\mathrm{d} E$.

For numerical simulation, the time increments $d=$ $1 / 50 f$ and $d=\tau / 25$ were used for SWV and SCV, respectively.

\section{Theoretical results}

The voltammetric response of a surface catalytic electrode mechanism is controlled mainly by the kinetics of the catalytic reaction and the kinetics of the heterogeneous electron transfer process. These two effects are represented by the catalytic parameter $\gamma$ and the kinetic parameter $\lambda$. According to definitions of these parameters $\left(\gamma=k_{\mathrm{c}} \tau, \lambda=k_{\mathrm{s}} \tau\right.$ for $\mathrm{SCV}$, and $\lambda=k_{\mathrm{s}} / f$, $\gamma=k_{\mathrm{c}} / f$ for SWV), one concludes that the overall influence of the catalytic and redox reactions is determined by the corresponding rate constant and the time window of the experiment, given by parameters $\tau$ or $f$.

Figure 1 shows the influence of the catalytic reaction on the shape of the SWV (A) and SCV (B) response. The increased rate of the catalytic reaction strongly affects the shape of both the forward and backward component of the SW voltammograms, but without a significant influence on the net SWV response (see Fig. 1A). Under high rates of the catalytic reaction, the backward and forward components gain a sigmoidal form, which is typical for a steady-state redox process. Nevertheless, the net component is still a well-defined peak with an easily measurable peak current, potential, and halfpeak width. This behaviour is apparently a remarkable benefit of SWV from both kinetic and analytical points of view.

The shape of the SCV response is strongly sensitive to the rate of the catalytic reaction. Its variations are rather
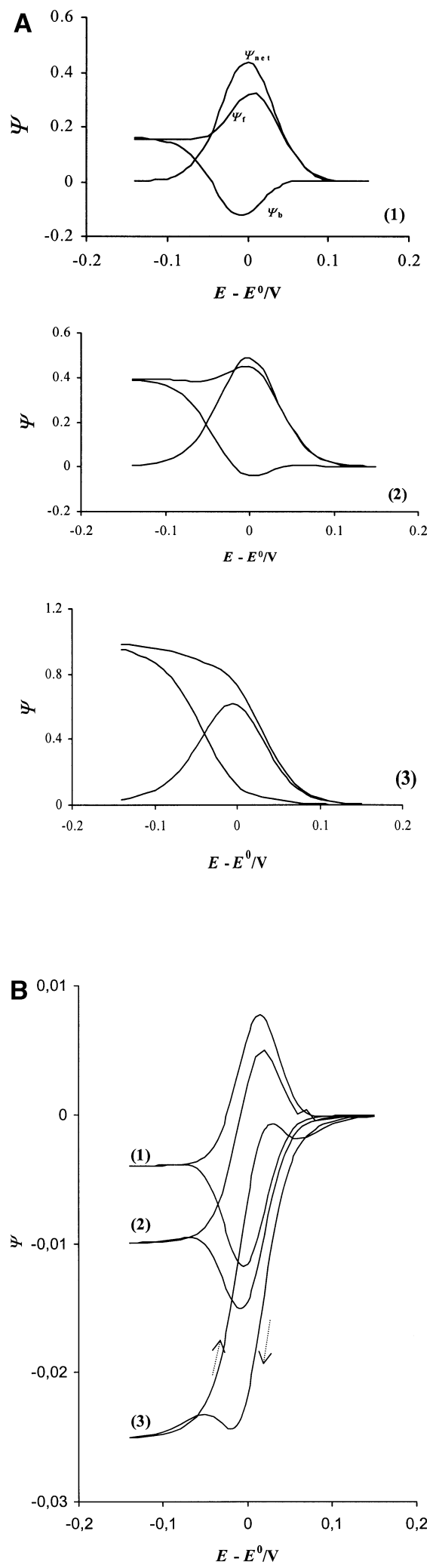

Fig. 1 Effect of the catalytic reaction on the dimensionless SWV (A) and SCV response (B). Conditions of the simulations in SWV were: $\lambda=2, n E_{\mathrm{sw}}=25 \mathrm{mV}, \mathrm{d} E=10 \mathrm{mV}, \alpha=0.5$, and $\log (\gamma)=$ $-0.8(1),-0.4$ (2), and 0 (3). Conditions of the simulations in SCV were: $\lambda=1, \mathrm{~d} E=10 \mathrm{mV}, \alpha=0.5$, and $\log (\gamma)=-2.4(1),-2(2)$, and $-1.6(3) . \Psi_{\mathrm{f}}, \Psi_{\mathrm{b}}$, and $\Psi_{\text {net }}$ are symbols for the forward, backward, and net component of the dimensionless SW response 
typical for this mechanism, which enables one to easily recognize a surface catalytic reaction in experiments. The increased current plateau at the switching potential, together with the increased cathodic and decreased anodic peak currents, are the most notable properties of the SCV response under influence of the catalytic reaction. Variations of the cyclic voltammograms become noticeable at very low rates of the catalytic reaction, which is not the case for SWV. However, for high catalytic rates, the situation is totally opposite. When the steady-state conditions are reached, a sigmoidal shape of the cyclic voltammograms appears (see Fig. 1B), whereas the SW voltammogram still has a well-defined peak (Fig. 1A). Obviously, both techniques complement each other.

Regardless of the applied technique, the catalytic reaction influences predominantly the peak currents, while the position of the response on the potential axis remains unchanged. Thus, Fig. 2 illustrates a more detailed analysis of the peak currents as functions of the catalytic parameter, for different reversibilities of the redox reaction. In SWV, the net SW peak current becomes sensitive to the catalytic reaction if $\gamma \geq 0.15$. For both irreversible $(\lambda=0.1$ in Fig. $2 \mathrm{~A})$ and reversible $(\lambda$ $=2$ in Fig. $2 \mathrm{~A}$ ) redox reactions, the peak current depends linearly on the catalytic parameter $\gamma$. Only within the quasi-reversible region $(\lambda=1$ in Fig. $2 \mathrm{~A})$ deviations from linearity occur, owing to the specific chronoamperometric features of the quasi-reversible redox reaction. Consequently, the quasi-reversible region will be addressed in more detail in the following text.

In SCV the ratio between the cathodic and anodic peak currents appears to be the most responsive criterion to the variation of the catalytic parameter. It is important to emphasize here that the ratio $\Psi_{\mathrm{p}, \mathrm{c}} / \Psi_{\mathrm{p}, \mathrm{a}}$ begins to be affected by the catalytic reaction for $\gamma \geq 0.001$. The limiting value of the catalytic parameter is almost two orders of magnitude lower than that of the corresponding one in SWV, which makes SCV a favourable technique to access low-rate catalytic processes. The upper limit of the catalytic parameter, at which the cyclic voltammogram still consists of measurable peaks, is about $\gamma=0.06$. This value is considerably lower than the upper limit measurable with SWV. This confirms the previous conclusion that SWV is the technique of choice for kinetic studies of fast catalytic reactions, as well as for analytical applications.

As mentioned at the beginning of this section, the voltammetric response of the electrode reaction studied depends also on the charge transfer kinetics, which is illustrated by the redox kinetic parameters $\lambda=k_{\mathrm{s}} / f$ and $\lambda=k_{\mathrm{s}} \tau$ for SWV and SCV, respectively. Figure 3 shows how the dimensionless SW peak currents (Fig. 3A), as well as the cathodic and anodic peak currents in SCV (Fig. 3B), vary with the redox kinetic parameters. At a moderate rate of the catalytic reaction, $\log (\gamma) \leq 0.1$, the dimensionless SW peak current depends parabolically on the redox kinetic parameter, forming a sharp maximum within the quasi-reversible region, i.e. $-1 \leq \log (\lambda) \leq 1$. This specific phenomenon is named as a "quasi-reversible maximum", which is a well-known feature accompanying all electrode reactions, in which at least one of the species of the redox couple is confined to the electrode surface [2, 18, 19]. The quasi-reversible maximum emerges owing to the specific chronoamperometric properties of the surface redox reaction, as well as the specific method of the current sampling procedure used in SWV [2,3]. When the rate of the redox reaction is synchronized to the frequency of the SW signal, then the highest current is measured at the end of each potential pulse. The origin and the attributes of the quasi-reversible maximum have been elaborated in detail in previous publications $[2,3,18]$. Owing to the strong influence of the redox kinetics on the voltammetric response within the quasi-reversible
Fig. 2 The effect of the catalytic parameter on the dimensionless peak currents in SWV (A) and SCV (B) for different values of the redox kinetic parameter. Conditions of the simulations in SWV were: $n E_{\mathrm{sw}}=20 \mathrm{mV}, \mathrm{d} E=5 \mathrm{mV}$, and $\alpha=0.5$. Conditions of the simulations in SCV were: $\mathrm{d} E=10 \mathrm{mV}$ and $\alpha=0.5 . \Delta \Psi_{\mathrm{p}}$ is a symbol for the dimensionless SW peak current, and $\Psi_{\mathrm{f}, \mathrm{a}}$ and $\Psi_{\mathrm{f}, \mathrm{c}}$ are the dimensionless anodic and cathodic peak currents in SCV, respectively
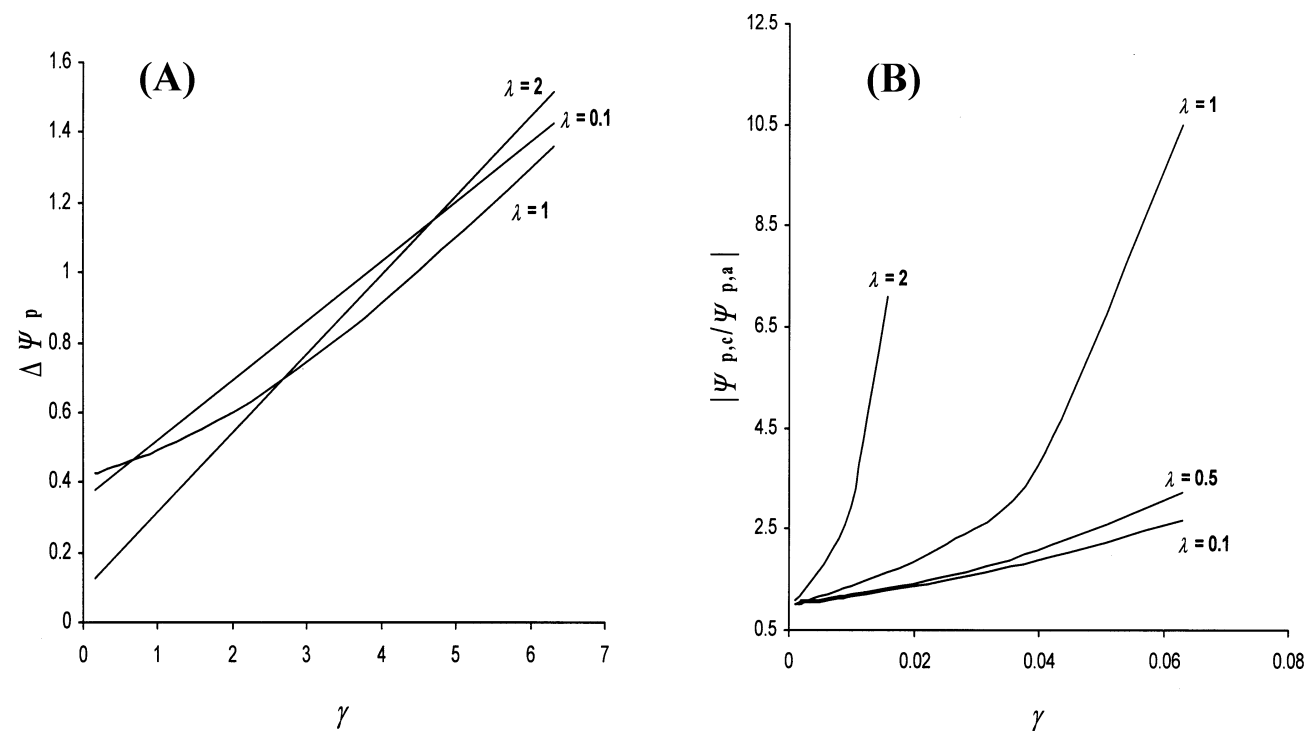
Fig. 3 The effect of the redox kinetic parameter on the dimensionless peak currents in SWV (A) and SCV (B) for different values of the catalytic parameter. Conditions of the simulations in SWV were: $n E_{\mathrm{sw}}=20 \mathrm{mV}, \mathrm{d} E=5 \mathrm{mV}$, and $\alpha=0.5$. Conditions of the simulations in SCV were: $\mathrm{d} E=10 \mathrm{mV}$ and $\alpha=0.5$

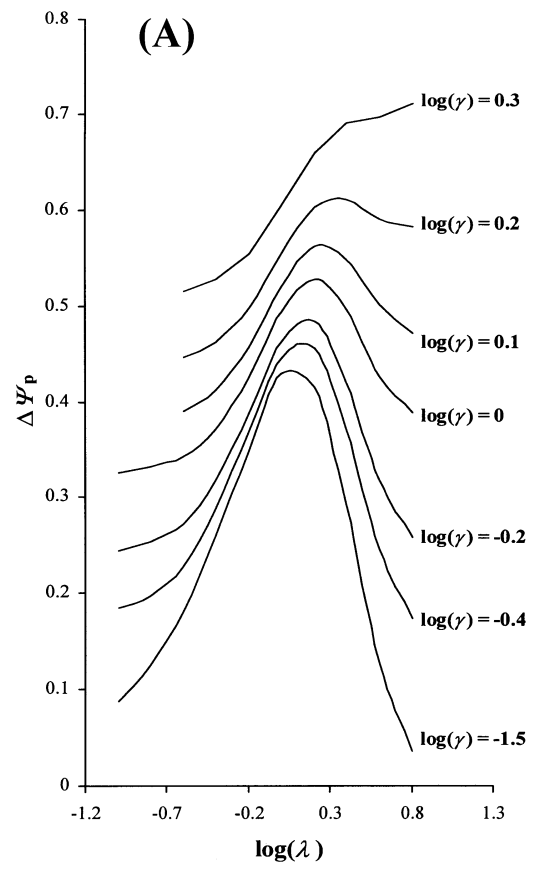

(B)

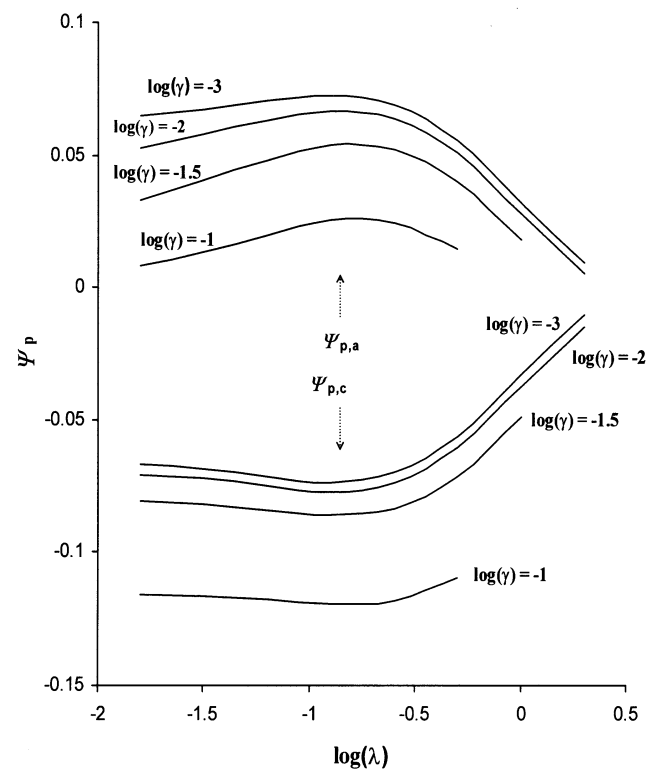

region, the SW peak current depends non-linearly on the catalytic parameter, as shown in Fig. 2A (see curve for $\lambda=1$ ).

When the rate of the catalytic reaction affects appreciably the overall kinetics of the redox mechanism, then the quasi-reversible maximum begins to lose its peak shape (Fig. 3A curve for $\log (\gamma)=0.2$ ). Finally, when the catalytic reaction is fast enough to transpose the entire electrode mechanism in steady-state conditions, the quasi-reversible maximum vanishes totally (Fig. 3A curve for $\log (\gamma)=0.3$ ). It is important to note, however, that as long as the quasi-reversible maximum exists, its position is independent of the particular value of the catalytic parameter (see Fig. 3A). This finding is of particular importance since it clearly indicates that the quasi-reversible maximum is a property controlled exclusively by the redox kinetics. Consequently, this phenomenon may be exploited for assessment of the standard rate constant of the surface catalytic redox reaction according to the methodology developed for simple surface redox reactions and elaborated in previous studies $[4,19]$.

Interestingly, owing to the phenomenon of the quasireversible maximum, the dependencies between both the cathodic and anodic peak currents and the kinetic parameter $\lambda$ are also non-linear in SCV (Fig. 3B). Note that this specific behaviour of the dimensionless peak currents in SCV is the crucial difference between the corresponding relationships in cyclic voltammetry with a linear potential scan $[8,9]$. However, in comparison to $\mathrm{SWV}$, the quasi-reversible maximum is not that well formed and its position cannot be determined with a satisfactory precision.

In the real experiment, when a single redox reaction is investigated, the latter analysis cannot be performed.
The reasons are that the redox kinetic parameter $\lambda(\lambda=$ $k_{\mathrm{s}} / f$ for SWV and $\lambda=k_{\mathrm{s}} \tau$ for SCV) may be altered experimentally only by varying the frequency of the SW signal, or duration of the potential tread in SCV. However, the alteration of $f$ or $\tau$ produces variations of the catalytic parameters also $\left(\gamma=k_{\mathrm{c}} / f\right.$ for SWV and $\gamma=$ $k_{\mathrm{c}} \tau$ for $\left.\mathrm{SCV}\right)$. Therefore, in order to approach to the experimental situation as close as possible, in the following the effect of $f$ and $\tau$ will be considered theoretically.

It is reasonable to expect that $f$ and $\tau$ exhibit a more complex influence on the voltammetric response, in comparison with the catalytic or kinetic parameters. The theoretical results in Fig. 4 show that the phenomenon of the quasi-reversible maximum can be achieved by varying the signal frequency $f$ or the duration of the potential tread $\tau$, if the rate of the catalytic reaction is appreciably slower than the rate of the redox transformation. In SWV, the overall kinetics of the electrode process is controlled by the redox kinetics if $k_{\mathrm{s}} \geq 6.667 k_{\mathrm{c}}$, where $k_{\mathrm{s}}$ and $k_{\mathrm{c}}$ are the standard redox rate constant and the catalytic rate constant, respectively. Under these conditions, the quasi-reversible maximum is well formed

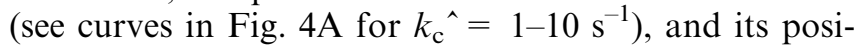
tion may be used to estimate the standard rate constant. If the relation between the kinetic constants is $k_{\mathrm{s}} \geq 2 k_{\mathrm{c}}$, then the influence of the catalytic reaction is predominant and thus the electrode process is transposed in steady-state conditions. Consequently, the relation between SWV peak currents and the signal frequency is sigmoidal without a defined maximum (see curves in Fig. 4A for $\left.k_{\mathrm{c}}=150-200 \mathrm{~s}^{-1}\right)$. In the intermediate region $\left(0.5 \leq k_{\mathrm{c}} / k_{\mathrm{s}} \leq 0.15\right)$, a mixed and complex kinetic control exists and both the redox and catalytic reactions control the voltammetric response (see curves in Fig. 4A 
for $\left.k_{\mathrm{c}}=50-100 \mathrm{~s}^{-1}\right)$. Within this region, the position of the quasi-reversible maximum depends on the catalytic reaction and it cannot be utilized for elucidation of the redox kinetics.

The results presented in Fig. 4B show also that the quasi-reversible maximum can be achieved by varying the duration of the potential tread in SCV. However, since the response in SCV is substantially more sensitive to the catalytic reaction, the quasi-reversible maximum appears only within the interval $k_{\mathrm{s}} \geq 11.11 k_{\mathrm{c}}$. Keeping in mind that the corresponding relation for SWV is $k_{\mathrm{s}} \geq 6.667 k_{\mathrm{c}}$, it is quite clear that the interval of accessible $k_{\mathrm{s}}$ values utilizing $\mathrm{SCV}$ is rather narrow in comparison to SWV.

Finally, it should be pointed out that the performance of the voltammetric response in SWV might be improved by adjusting the signal amplitude, which appears to be an additional advantage in this technique. Figure 5 depicts how the SW amplitude increases the sensitivity of the peak currents to the rate of the catalytic reaction. Obviously the higher the signal amplitude, the higher is the sensitivity of the peak currents to the rate of the catalytic reaction, which is of particular importance for analytical purposes. However, the SW amplitude cannot be increased much, since for amplitudes higher than approximately $100 \mathrm{mV}$ the response splits into two peaks. The splitting of the response at large signal amplitudes is a general property of surface redox reactions that was elaborated in our previous communications $[6$, 24]. It appears as a consequence of the large potential separation between the forward and backward components of the SWV response. This unique phenomenon of
Fig. 4 The effect of the frequency and duration of the potential treads on the dimensionless peak currents in SWV (A) and SCV (B) for different rates of the catalytic reaction. Conditions of the simulations in SWV were: $n E_{\mathrm{sw}}=20 \mathrm{mV}$, $\mathrm{d} E=5 \mathrm{mV}, \alpha=0.5$, and $k_{\mathrm{s}}=316 \mathrm{~s}^{-1}$. Conditions of the simulations in SCV were: $\mathrm{d} E=10 \mathrm{mV}, \alpha=0.5$, and $k_{\mathrm{s}}=10 \mathrm{~s}^{-1}$
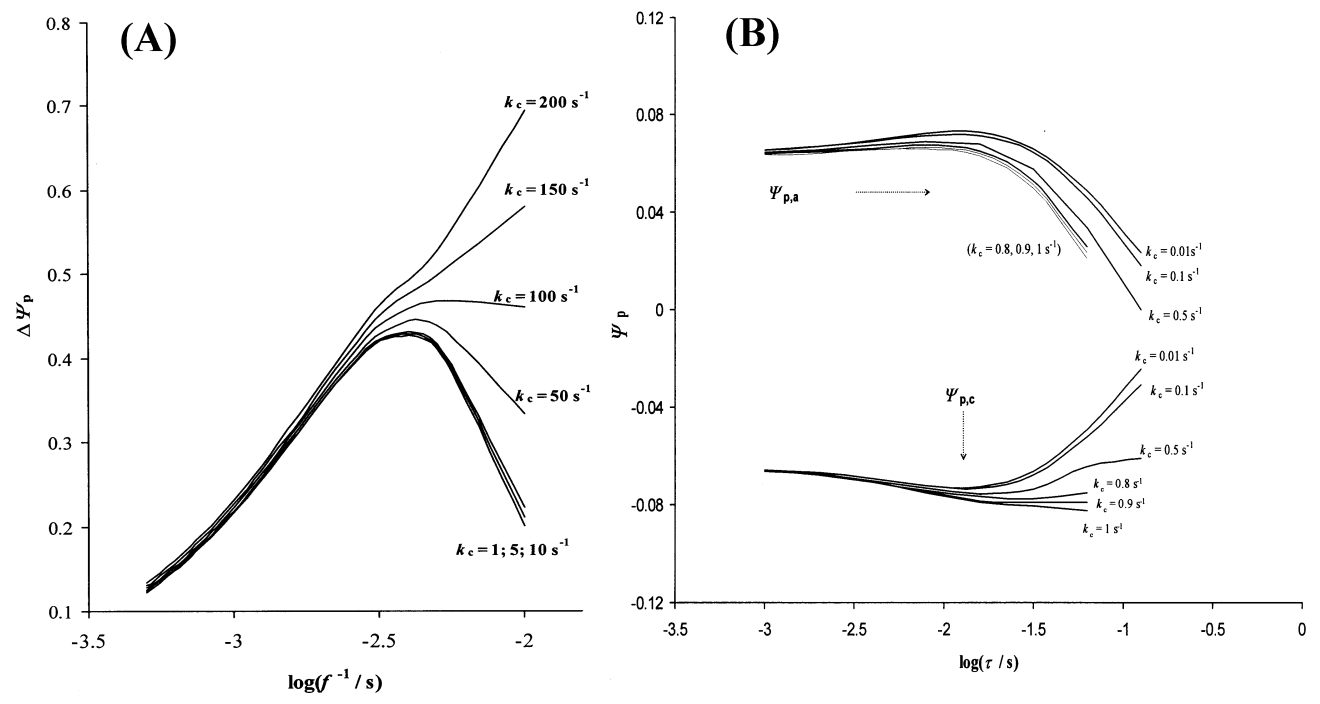

Fig. 5 The effect of the catalytic parameter on the dimensionless SWV peak currents for different signal amplitudes. Conditions of the simulations were: $\mathrm{d} E=5 \mathrm{mV}, \lambda=2$, and $\alpha=0.5$

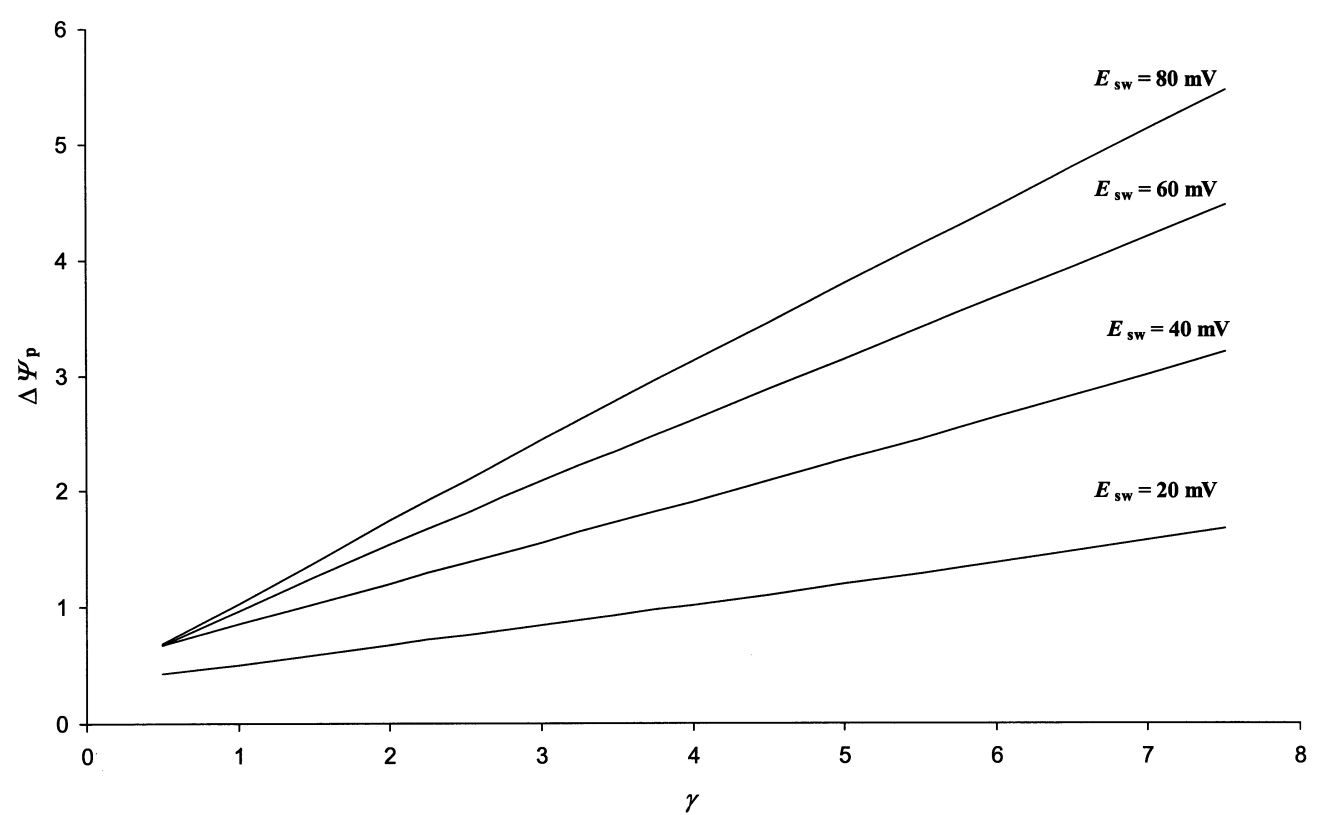


surface redox processes can be utilized for a complete kinetic characterization of simple surface redox reactions; however, from an analytical point of view, it is completely undesirable. Moreover, the split SW peaks in the presence of the catalytic reaction behave in a rather complex way, and generally speaking this phenomenon provides no useful information in the case of surface catalytic redox reactions.

\section{Experimental}

All used chemicals were of analytical reagent grade (Merck). Redistilled water was used. Acetate buffer solutions $(0.1 \mathrm{~mol} / \mathrm{L})$ were used as supporting electrolytes. A stock solution of hydrogen peroxide with a concentration of $8 \%(\mathrm{v} / \mathrm{v})$ was used. The amounts of hydrogen peroxide added to the electrolyzed solutions are indicated on the figures. A stock solution of azobenzene was prepared by dissolving in glacial acetic acid. Extra pure nitrogen was used for purging the electrolyte solutions for $10 \mathrm{~min}$ prior to each measurement. A nitrogen blanket, over the electrolyte solution, was maintained thereafter.

All voltammograms were recorded using a $\mu$ Autolab (ECO Chemie, Utrecht, Netherlands), which was connected to a static mercury drop electrode (SMDE) (model 303A, Princeton Applied Research). A platinum wire was used as the auxiliary electrode and $\mathrm{Ag} / \mathrm{AgCl} / \mathrm{KCl}(3 \mathrm{~mol} / \mathrm{L})$ as the reference electrode. The measurements were carried out at room temperature.

\section{Results}

Azobenzene/hydrazobenzene is the well-known redox couple in which both components of the couple are strongly adsorbed on a mercury electrode surface $[3,35$, $36,37]$. The redox transformation in an aqueous medium involves chemically reversible exchange of two electrons and two protons. This redox reaction is used widely for illustrations of different theoretical models, such as surface redox processes [4, 24, 35, 37], and electrode reactions coupled with adsorption phenomena in general [18].

In this work, the redox reaction of azobenzene was investigated in the presence of hydrogen peroxide as oxidizing agent. Hydrazobenzene, formed by electrochemical reduction, is reoxidized to azobenzene by hydrogen peroxide, and the whole electrode reaction turns into a surface catalytic mechanism (Eqs. 11 and 12):

$\mathrm{Ph}-\mathrm{N}=\mathrm{N}-\mathrm{Ph}+2 \mathrm{e}^{-}+2 \mathrm{H}^{+} \leftrightarrows \mathrm{Ph}-\mathrm{NH}-\mathrm{NH}-\mathrm{Ph}$

$\mathrm{Ph}-\mathrm{NH}-\mathrm{NH}-\mathrm{Ph}+\mathrm{H}_{2} \mathrm{O}_{2} \leftrightarrows \mathrm{Ph}-\mathrm{N}=\mathrm{N}-\mathrm{Ph}+2 \mathrm{H}_{2} \mathrm{O}$

All the experiments were carried out in acetate buffer at $\mathrm{pH}$ 3.8. In this medium, for small $\mathrm{SW}$ amplitudes $\left(E_{\mathrm{sW}}<100 \mathrm{mV}\right)$ the $\mathrm{SW}$ response of azobenzene consists of a well-developed peak with a peak potential of about $-0.24 \mathrm{~V}$ vs. $\mathrm{Ag} / \mathrm{AgCl}$. In SCV, the response consists of a pair of peaks, located symmetrically with respect to the potential axis, and with almost equal peak heights.
Increasing the accumulation time caused both peaks of the cyclic voltammogram to increase proportionally. Moreover, when the electrode surface is saturated with the electroactive material, the subsequent cycling of the potential exhibited no significant influence on the response, and steady-state voltammograms were obtained. All these results indicate that the electrode reaction of azobenzene in this medium proceeds predominantly as a surface redox process, which is the main prerequisite for illustration of the theoretical results.

In the presence of hydrogen peroxide as oxidizing agent, the simple surface redox reaction is transformed into the surface catalytic mechanism. The rate of the catalytic reaction is controlled by adjusting the concentration of oxidizing agent. When the concentration of hydrogen peroxide is increased from 0 to $2.4 \%(\mathrm{v} / \mathrm{v})$, then the SWV response is enhanced, while its position and shape remain virtually unaffected (see Fig. 6). Under the same experimental conditions, the diversity of the cyclic voltammetric response is more evident (see Fig. 7). The current plateau at the switching potential is strongly raised owing to the presence of the oxidizing agent. Furthermore, at $2.4 \%(\mathrm{v} / \mathrm{v})$ concentration of $\mathrm{H}_{2} \mathrm{O}_{2}$, the experiment is close to the steady-state conditions, and consequently the shape of the cyclic voltammogram changes severely (see Fig. 7). It should be noted that the current is also increased before reaching the reduction potential of azobenzene, which was not predicted by the theoretical results. This phenomenon is most probably a consequence of a partial faradic activity of the oxidizing agent itself. Nevertheless, it is important to note that under the same experimental conditions the SWV response still persists in the catalytic reaction, which is in agreement with the theoretical predictions (see Fig. 1).

A more comprehensive analysis of the effect of an increasing concentration of the catalytic agent on the

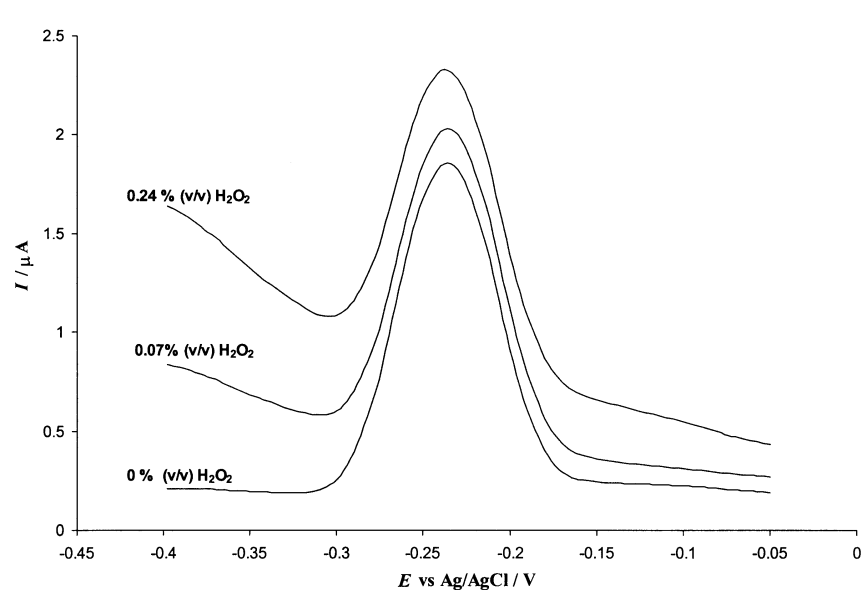

Fig. 6 The influence of hydrogen peroxide on the SWV response of a $5 \times 10^{-6} \mathrm{~mol} \mathrm{~L}^{-1}$ solution of azobenzene recorded in acetate buffer at $\mathrm{pH}$ 3.8. Other experimental conditions were: $f=35 \mathrm{~Hz}, E_{\mathrm{sw}}=$ $20 \mathrm{mV}, \mathrm{d} E=4 \mathrm{mV}, t_{\mathrm{acc}}=5 \mathrm{~s}$, and $E_{\text {acc }}=-0.05 \mathrm{~V}$, where $t_{\text {acc }}$ is the accumulation time and $E_{\text {acc }}$ is the accumulation potential 

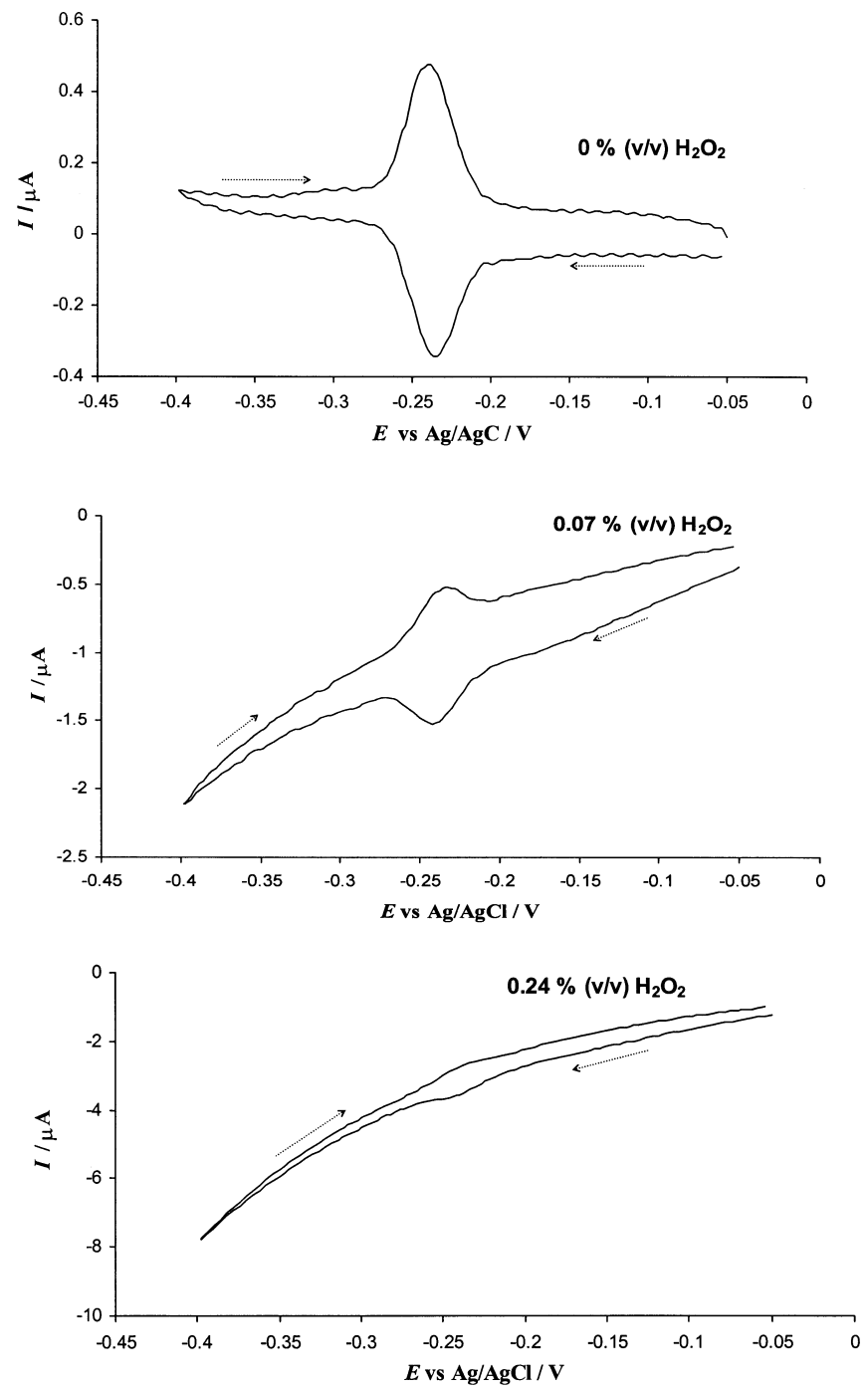

Fig. 7 The influence of hydrogen peroxide on the SCV response of a $5 \times 10^{-6} \mathrm{~mol} \mathrm{~L}^{-1}$ solution of azobenzene recorded in acetate buffer at $\mathrm{pH}$ 3.8. Other experimental conditions were: $\mathrm{d} E=4 \mathrm{mV}, \tau=$ $0.033 \mathrm{~s}, t_{\mathrm{acc}}=5 \mathrm{~s}$, and $E_{\mathrm{acc}}=-0.05 \mathrm{~V}$

peak currents, in both SWV and SCV, is demonstrated in Fig. 8. The experimental analysis is undertaken at different frequencies and lengths of the potential tread, which corresponds to the theoretical study in which the influence of the catalytic parameter at a distinct reversibility of the redox reaction is investigated (cf. Figs. 2 and 8). Evidently, for both techniques the shapes of the experimental dependencies resemble the theoretical ones, supporting the predictions of the theory. At frequencies of 35 and $60 \mathrm{~Hz}$ the redox reaction is still in the reversible region, which causes the relation between the SWV peak currents and concentration of hydrogen peroxide to be linear (see Fig. 8A). At a frequency of $100 \mathrm{~Hz}$, the corresponding relationship is non-linear due to the quasi-reversibility of the redox reaction.

Finally, the effect of the SW signal frequency and the length of the potential tread in SCV will be briefly discussed. Varying the frequency from 10 to $120 \mathrm{~Hz}$ resulted in the appearance of the quasi-reversible maximum of azobenzene (Fig. 9A). Note that the ratios $\Delta I_{\mathrm{p}} / f$ and $1 / f$ correspond to the dimensionless peak current $\Delta \Psi_{\mathrm{p}}=\Delta I_{\mathrm{p}} / n F S f \Gamma_{0}$ and redox kinetic parameter $\lambda$ $=k_{\mathrm{s}} / f$, respectively. The quasi-reversible maximum is positioned at about $100 \mathrm{~Hz}$, implying that the standard rate constant of azobenzene under these conditions is close to the value of $100 \mathrm{~s}^{-1}$ [4]. Here, it is important to emphasize that the position of the quasi-reversible maximum does not vary significantly in the presence of the catalytic agent. This experimental finding supports
Fig. 8 The effect of hydrogen peroxide on the peak currents $I_{\mathrm{p}}$ in SWV (A) and SCV (B). The SWV frequencies and duration of the potential tread are indicated on the graphs. The experimental conditions were: $c(\mathrm{azo})=5 \times 10^{-6} \mathrm{~mol} \mathrm{~L}^{-1}$ in $1 \mathrm{~mol} \mathrm{~L}^{-1}$ acetate buffer at $\mathrm{pH} 3.8, t_{\mathrm{acc}}=5 \mathrm{~s}, f=35 \mathrm{~Hz}$, $E_{\mathrm{sw}}=20 \mathrm{mV}$, and $\mathrm{d} E=4 \mathrm{mV}$
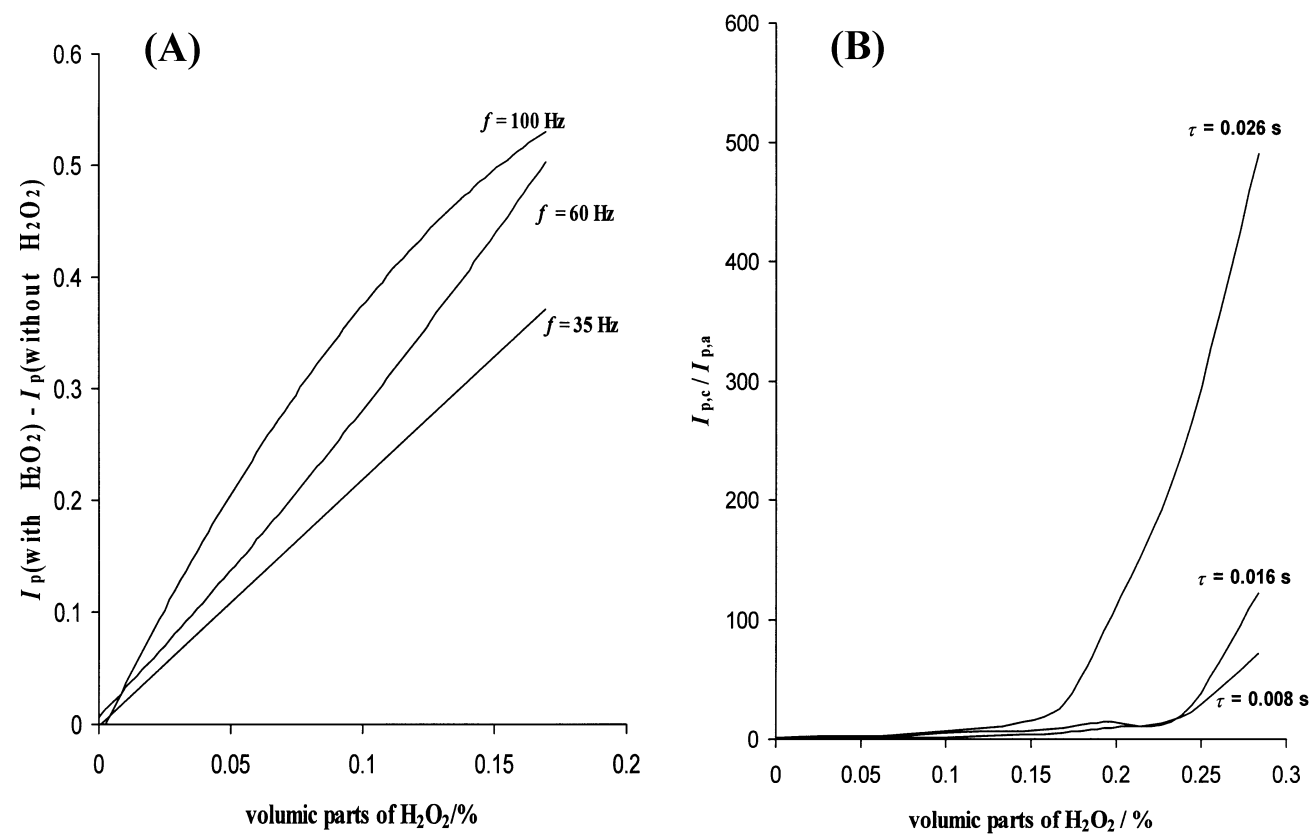
Fig. 9 The quasi-reversible maximum of azobenzene in SWV (A) and SCV (B) measured in the presence of different amounts of hydrogen peroxide. The experimental conditions were the same as in Fig. 8

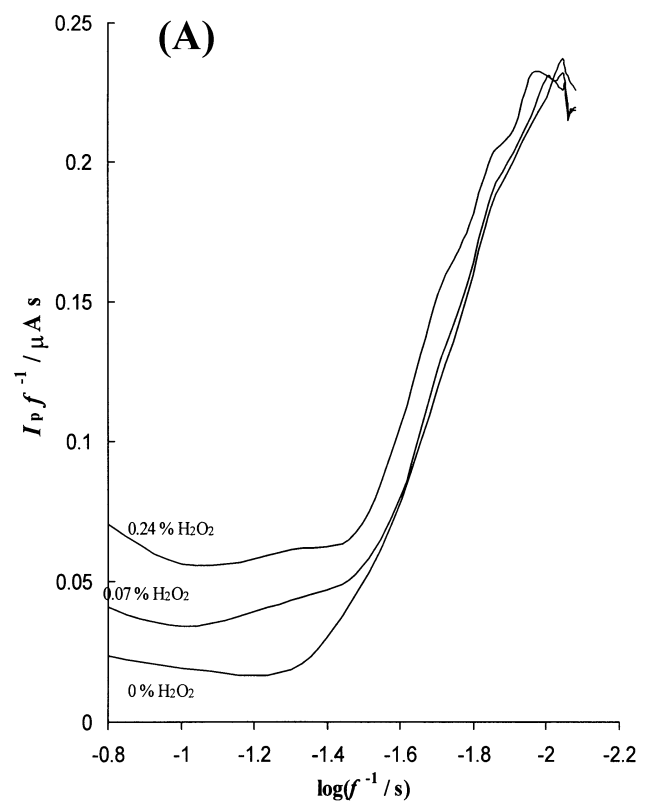

the conclusion derived theoretically that the quasire\#132;versible maximum is a feature which is exclusively controlled by the charge transfer kinetics (see the theoretical results in Figs. 3A and 4A).

Although poorly defined, the quasi-reversible maximum is also demonstrated by the experiments in SCV (Fig. 9B). It is better noticeable by analyzing the anodic peak currents, since the cathodic branches of the cyclic voltammograms are influenced severely by the catalytic reaction. Nevertheless, the superiority of SWV in the study of this feature is evident.

In summary, the agreement between the theoretical and experimental results confirms the validity of the presented theoretical model.

Acknowledgements V.M. thanks A.V. Humboldt-Stiftung for provision of a Humboldt fellowship and R.G. thanks Deutscher Akademischer Austauschdienst (DAAD) for provision of a Ph.D. scholarship. We thank Professor Fritz Scholz for useful discussions during the preparation of the manuscript.

\section{References}

1. Komorsky-Lovrić Š (1986) Mikrochim Acta 1:407

2. Lovrić M, Komorsky-Lovrić Š (1988) J Electroanal Chem 248:239

3. Komorsky-Lovrić Š, Lovrić M (1995) J Electroanal Chem $384: 115$

4. Komorsky-Lovrić Š, Lovrić M (1995) Anal Chim Acta 305:248

5. O'Dea JJ, Song S, Bowden EF (1993) Anal Chem 65:3090

6. Mirčeski V, Lovrić M (1997) Electroanalysis 9:1283

7. O'Dea JJ, Osteryoung J (1997) Anal Chem 69:650

8. Meunier-Prest R, Laviron E (1996) J Electroanal Chem 410:133

9. Laviron E (1979) J Electroanal Chem 101:19
10. Barker GC (1960) In: Advances in polarography; proceedings of the 2nd international congress, vol 1, p 144

11. Christie JH, Lingane P J (1965) J Electroanal Chem 10:176

12. Bilewicz R, Osteryoung RA, Osteryoung J (1986) Anal Chem $58: 261$

13. Saralathan M, Osteryoung RA, Osteryoung J (1986) J Electroanal Chem 214:141

14. Saralathan M, Osteryoung RA, Osteryoung J (1987) J Electroanal Chem 222:69

15. Penczek M, Stojek Z, Buffle J (1989) J Electroanal Chem 270:1

16. Kalapathy U, Tallman DE (1992) Anal Chem 64:2693

17. Komorsky-Lovrić S, Lovrić M (1996) Electroanalysis 8:959

18. Mirčeski V (2001) J Electroanal Chem 508:138

19. Mirčeski V, Gulaboski R, Jordanoski B, Komorsky-Lovrić S (2000) J Electroanal Chem 490:37

20. Webber A, Shah M, Osteryoung J (1984) Anal Chim Acta $157: 1$

21. Webber A, Osteryoung J (1984) Anal Chim Acta 157:17

22. Lovrić M, Komorsky-Lovrić S, Murray RW (1988) Electrochim Acta 33:739

23. O'Dea JJ, Ribes A, Osteryoung J (1993) J Electroanal Chem 345:287

24. Mirčeski V, Gulaboski R (2001) Electroanalysis 13:1326

25. Vega M, van den Berg GMG (1994) Anal Chim Acta 293:19

26. van den Berg GMG (1991) Anal Chim Acta 250:265

27. Bond AM, Luscombe DL (1986) J Electroanal Chem 214:21

28. Jiao K, Jin W, Metzner H (1992) Anal Chim Acta 260:35

29. Bartlett PN, Tebbutt P, Tyrrell CH (1992) Anal Chem 64:138

30. Mugweru A, Rusling JF (2001) Electrochem Commun 3:406

31. Hilditch PI, Green MJ (1991) Analyst 116:1217

32. Okuma H, Takahashi H, Sekimukai S (1991) Anal Chim Acta 244:161

33. Uchiyama S, Suzuki S, Sato T (1990) Electroanalysis 2:559

34. Nicholson RS, Olmstead ML (1972) In: Mattson JS, Mark HB, MacDonald HC (eds) Electrochemistry: calculations, simulation and instrumentation, vol. 2. Dekker, New York, pp 120 137

35. O'Dea JJ, Osteryoung J (1993) Anal Chem 65:3090

36. Komorsky-Lovrić Ś, Lovrić M (1995) Anal Chim Acta 305:248

37. Laviron E (1973) J Electroanal Chem 42:415 